\title{
Differential Distribution of NMDA and AMPA Receptor Activity in Layer II/III in Mouse Primary Visual Cortex
}

\author{
F.P. Bellinger* and T.K. Hensch**
}

*Békésy Laboratory of Neurobiology, Pacific Biosciences Research Center, University of Hawaii, 1993 East West Rd, Honolulu, HI, 96822, USA.

**Laboratory for Neuronal Circuit Development, RIKEN Brain Science Institute,

2-1 Hirosawa, Wakoshi, Saitama, 351-0198, Japan

The role of NMDA receptors in visual cortex plasticity has received much recent examination [1]. In order to locate specific regions of synapses that may be sensitive to NMDA receptor-mediated plasticity, this study compares the contribution of AMPA and NMDA activity to synaptic responses throughout primary visual cortex in response to layer IV stimulation by using voltage-sensitive dye imaging.

Optical absorbance signals of the voltage-sensitive dye RH482 in responses were imaged in the binocular region of mouse primary visual cortex slices using the Deltaron 1700 imaging system (Fujix, Japan) [2]. Each slice was sequentially imaged in normal artificial cerebral spinal fluid (ACSF), ACSF with the NMDA receptor antagonist AP5, and ACSF with both the AMPA receptor antagonist CNQX and AP5. Subtracting the images taken in the presence of AP5 from the images taken in normal saline revealed the NMDA receptor activity, and subtracting the CNQX/AP5 images from images in AP5 alone produced AMPA receptor images. Signals were quantified as percent changes in light from prestimulus values. AMPA and NMDA images from 7 visual cortex slices were compared by measuring pixels in peak images vertically through the center of activity or horizontally through the peak activity in layer II/III.

The AMPA receptor signal was strongest in the middle of layer II/III, $148 \pm 9 \mu \mathrm{m}$ from the cortical surface, at $6.0 \pm 0.2 \mathrm{~ms}$ after stimulation. In contrast, the NMDA receptor signal was strongest at the layer I/layer II border, with peak activity at $57 \pm 10 \mu \mathrm{m}$ from the surface, at $8.5 \pm 05 \mathrm{~ms}$ post-stimulus. The horizontal spread of the NMDA signal was more homogeneous, but the overall traveled distance was no different from AMPA responses.

These results show that NMDA and AMPA contributions to synaptic activity differ in their vertical location throughout the binocular region of mouse visual cortex. This may indicate different receptor compositions in synapses upon different cell types (i.e. cells with tufted vs. non-tufted dendrites, or interneurons vs. pyramidal neurons).

\section{References}

[1] T.K. Hensch and M. Fagiolini, Prog. Brain Res. 147 (2005) 115.

[2] M.Tanifuji, Brain Res. 738 (1996) 83.

[3] This research was supported by the Hawaii Community Foundation and the RIKEN Brain Science Institute, Japan. 

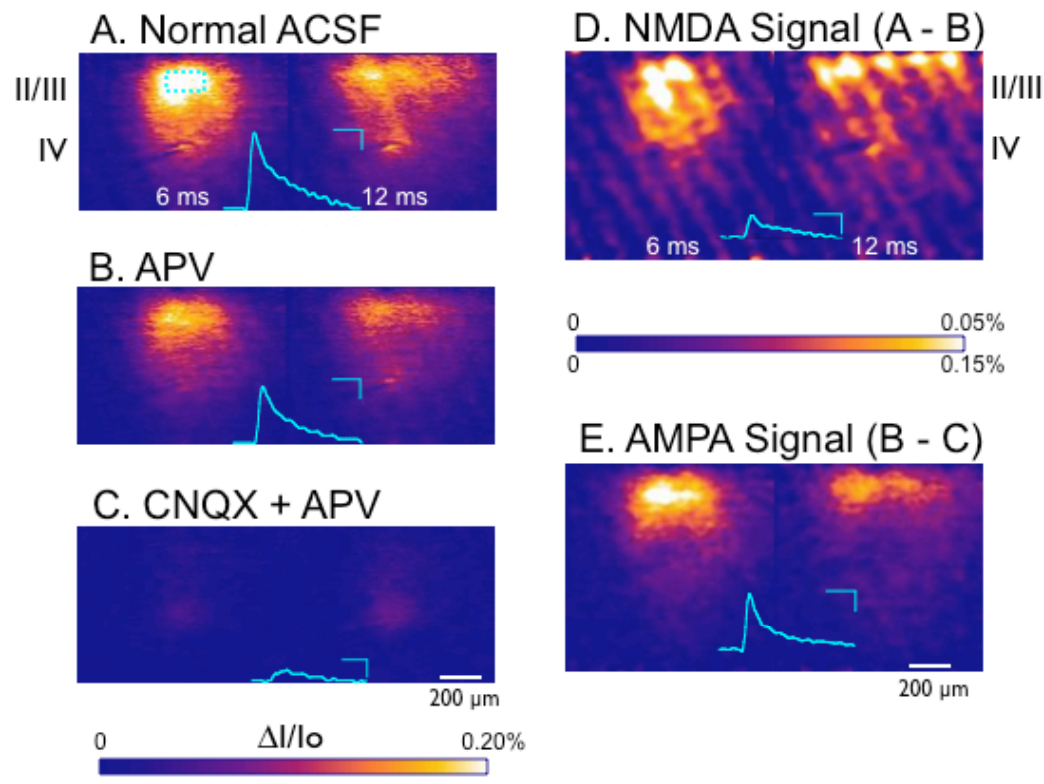

FIG. 1. Digital isolation of AMPA and NMDA receptor responses. A: Example of voltage-sensitive dye images in layer II/III at 6 and $12 \mathrm{~ms}$ post-layer IV stimulation of mouse visual cortex slice in normal ACSF. Superimposed traces indicate temporal profile of activity averaged over a $196 \mathrm{X} 98 \mu \mathrm{m}$ area (indicated by broken line) in layer II/III ( $0.05 \%$ change in light transmission, and horizontal: 25 ms). B: Same as A but in the presence of AP5. C. Same as B with CNQX as well as AP5. D: NMDA signal produced by subtracting image $B$ from A. E: AMPA signal revealed by subtracting $C$ from $B$.
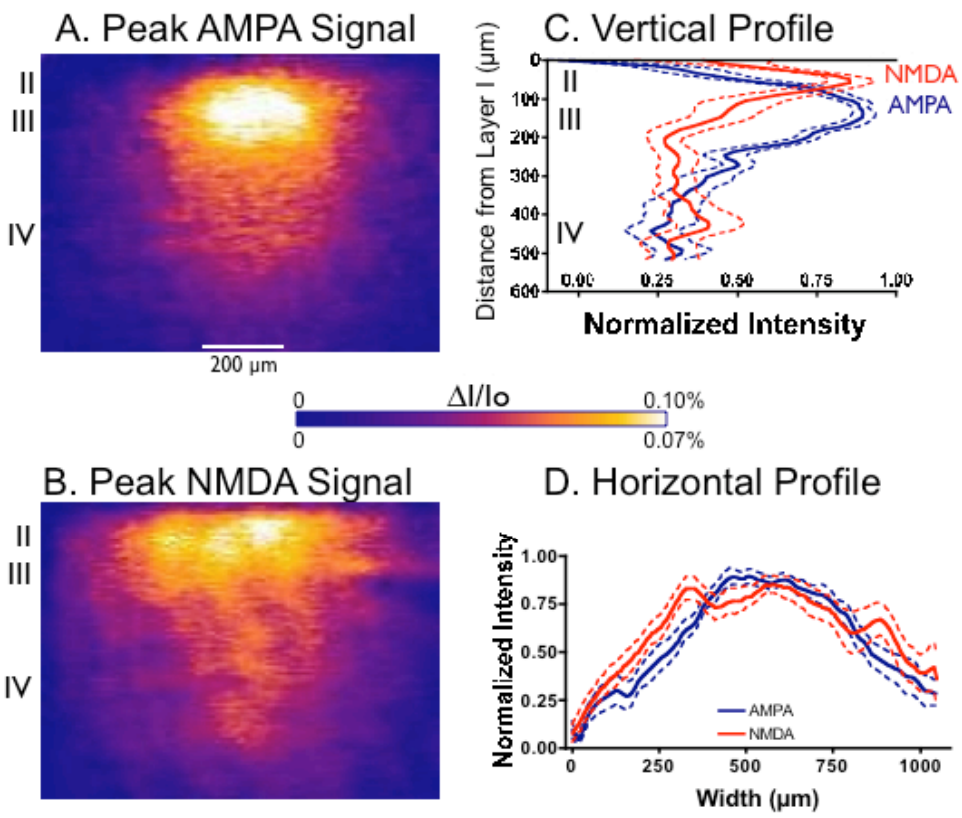

FIG. 2. Comparison of AMPA and NMDA peak responses. A: Arithmetic mean of AMPA signals from 7 slices at $6 \mathrm{~ms}$ post-stimulus. B: Arithmetic mean of peak NMDA signals from 7 slices $9 \mathrm{~ms}$ post-stimulus. C: Comparison of vertical signal profiles of AMPA and NMDA signals (solid line: mean, broken line: SEM). D: Horizontal profile through peak intensity changes in layer II/III for NMDA and AMPA signals. 